\title{
Sacrectomy as treatment of giant cell tumor in Dr. M. Djamil Hospital Padang: A Case Report
}

\author{
Roni Eka Sahputra ${ }^{1}$, Menkher Manjas ${ }^{2}$, Azdiana Fitri ${ }^{3}$, Septry Larissa ${ }^{4}$ \\ \{roni78esahputra@gmail.com ${ }^{1}$, menkherm@yahoo.com ${ }^{2}$, azfitri010110@gmail.com ${ }^{3}$, \\ septry.larissa@gmail.com $\left.{ }^{4}\right\}$ \\ Orthopaedic Division of Surgical Departement, Medical Faculty of Universitas Andalas/Dr. M.Djamil \\ Hospital Padang - West Sumatera ${ }^{1,2}$ \\ Surgical Departement, Medical Faculty of Universitas Andalas/Dr. M.Djamil Hospital Padang - West \\ Sumatera ${ }^{3,4}$
}

\begin{abstract}
Primary sacral tumors are rare, $7 \%$ of all spinal tumors. Most sacral tumors are benign aggressive lesions such as aneurysmal bone cyst, osteoblastoma, and giant cell tumor, or low-grade malignancies such as chordoma or chondrosarcoma. We report a case of Giant cell tumour of the sacrum in a female patient 59 years old noticed with pain in lower back with radiated to the left thigh with muscle weakness since 6 months. No complain about bowel habit and micturition. Coronal and sagittal non-contrast T1\&T2 MRI showed destructive heterogeneous intermediate-to-high signal densities within the lesion, indicating tumor localized at S1 to coccygeus.Total sacrectomy performed with exploration and preservation of major vessels by anterior approach following spinopelvic reconstruction procedure by posterior approach. Histopathologic examinations confirmed giant cell tumor.Total gastrectomy is a complex surgical procedure that requires specialized surgical stabilization techniques to preserve mechanical support and repair walking ability. Postoperative local recurrence rate after sacral tumor resection is directly related to the scope and degree of the resection. The risk of infection increases in patients who have previously undergone lumbosacral surgery and procedures with a long operation time.1Sacral tumors treated with total/partial sacrectomy and lumbopelvic stabilization show good functional outcomes and low complication rates.
\end{abstract}

Keywords: Giant Cell Tumour, Sacrectomy.

\section{Introduction}

Primary sacral tumors are rare, accounting for $7 \%$ of all spinal tumors. They can arise from the bone in the skull base and anywhere along the spine; however, the most common site is the sacrococcygeal region $(40 \%-50 \%)$ and the base of the skull $(35 \%-40 \%)$ followed by the vertebral bodies $(15 \%-20 \%)$ [1]-[2].

They can arise from a neoplastic nidus in bone, cartilage, or soft tissue (neural tissue, meningeal tissue, or embryonic rests). Most sacral tumors are benign aggressive lesions such as an aneurysmal bone cyst, osteoblastoma, and giant cell tumor, or low-grade malignancies such as chordoma or chondrosarcoma [1]-[2].

Giant cell tumors are locally aggressive, benign bone tumors with a high risk of local recurrence and a low risk of metastasis after treatment [1],[3]. They are most common in the second decade and more common in females than males. They typically affect the epiphyseal 
area of long bones. In the axial skeleton, the sacrum is the most common place of involvement by where Giant cell tumors (GCT) form. The incidence of GCT in the sacrum is between $6.7 \%$ to $9.4 \%$ in different series [1-4].

Tumors sacral region is usually diagnosed late and often present as large, advanced neoplastic masses because of mild initial symptoms. The local recurrence rate of sacral tumors is relatively high due to complex anatomy and advanced disease. Intralesional curettage, following by cryo or thermal cauterization may produce a complete cure for benign lesions. In contrast, wide resection is necessary for the cure or long-term disease-free control in radio- and chemo-resistant malignancies [1].

Sacral GCT has a high local recurrence tendency compared to other skeletal regions. GCT sacral recurrence rates are reported to reach $33 \%$. The act of enbloc resection is an option to reduce the recurrence. At present, the intra-lesion resection is followed by inactivation of intracavitary lesions preferred by doctors and patients compared to en bloc resection due to their complications. Variations in the choice of adjuvant therapy after intralesional resection are carried out much like intracavitary cryosurgery, radiotherapy, chemotherapy, and selective arterial embolization. If removal of the entire tumor is not possible, intralesional curettage can be done even with a high risk of recurrence. Several studies have recommended intraoperative cryosurgery as an adjuvant. The action is much successful as a therapy for GCT in the long bones, but the success of sacrum is still not widely reported. Difficulty factors to get a wide margin of excision such as large tumor size, pelvic anatomy, neurovascular structure [1]-[2].

Large tumor size becomes a complication of surgery so that it will require a long operating time. Blood loss and wound complications are considered in the act of removing the tumor. Resection of large tumors causes instability of the lumbopelvic region and threatens the patient's neurological function. Partial sacectomy can be performed without the need for lumbopelvic stabilization, but patients with extensive tumors need total sacrectomy which results in vertical instability and rotation, requiring stabilization measures for mobilization and increased neurologic function. The extent of sacral resection influences outcomes and the are of the amputation, mechanical and neurological complications, the resection margins, and the possible occurrence of local and systemic complications [1],[3],[4].

\section{Case Report}

The patient is a 59-year-old previously healthy woman who presented with pain in lower back with radiated to the left thigh to toe with muscle weakness since 6 months ago. There is no complaint about bowel habit and micturition. On examination, he had neurological deficits and had tenderness to palpation over the distal sacrum and coccyx. Coronal and sagittal non-contrast T1 and T2 MRI images showed destructive heterogeneous intermediate-to-high signal densities within the lesion, indicating tumor localized at the $\mathrm{S} 1$ to coccygeus (Figure 1). The patient was taken to the operating room in conjunction with colorectal and vascular surgery specialists. We did total sacrectomy and en bloc resection of the lesion. Total sacrectomy was performed via the posterior approach along with exploration and preservation of major vessels ( internal iliaca artery bilateral ) by the anterior approach and following with spinopelvic reconstruction procedure by posterior approach (Figure $2 \& 3$ ). The stabilization is achieved with 2 rods in order to provide stable fixation (Figure 4). Intraoperative bleeding was about $4.500 \mathrm{ml}$. The patient tolerated the procedure well and was discharged home on postoperative day 5 . Histopathologic examinations confirmed as giant cell tumor with negative surgical margins. 
Given that there was en bloc resection of the tumor with negative margins, the patient was then just observed every 3 months for local recurrence prevention. No local recurrence was determined during the 3 months follow-up period. At her three months follow-up, the patient was doing extremely well with resolved pain, a well-healed incision, but with neurological deficits of bowel and bladder.

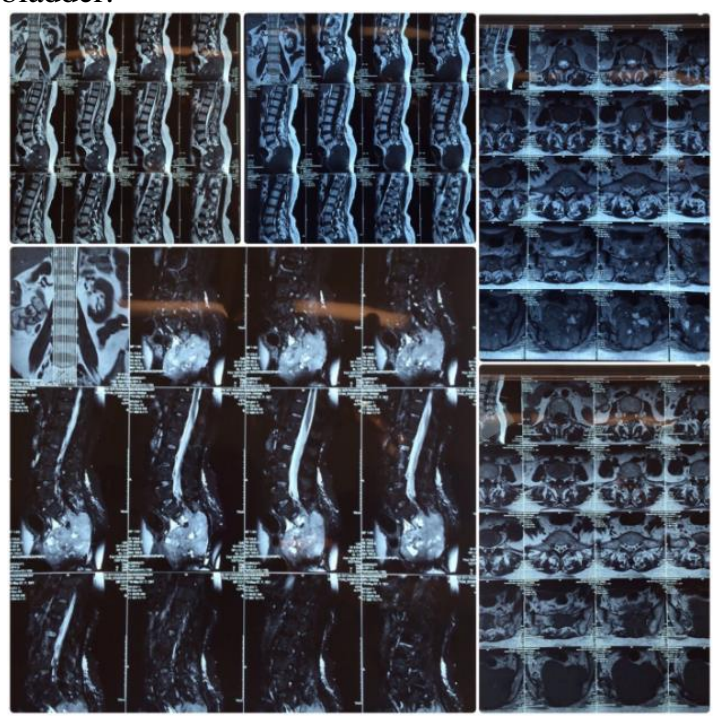

Figure 1. Preoperative Imaging, Coronal and sagittal non-contrast T1 and T2 MRI images showed destructive heterogeneous intermediate-to-high signal densities within the lesion, indicating tumor localized at the $\mathrm{S} 1$ to coccygeus

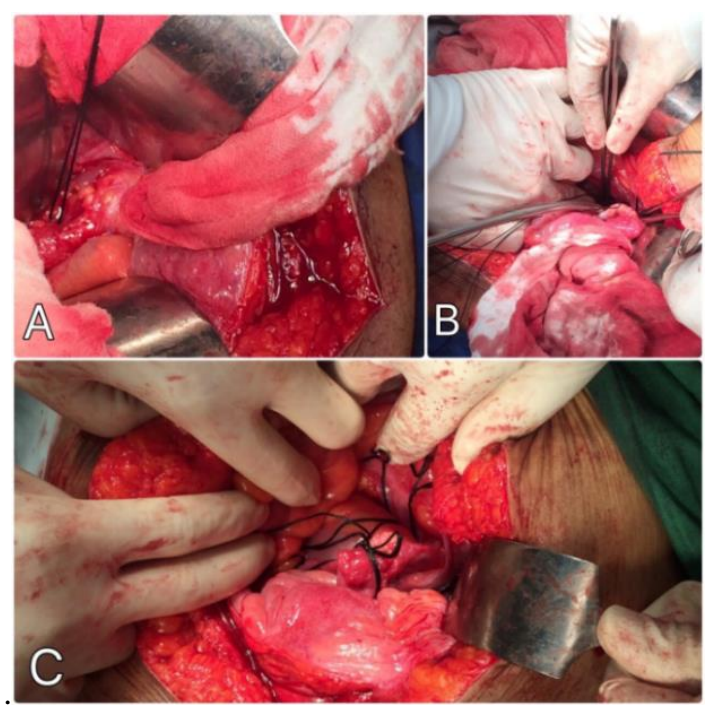

Fig. 2 : Ligation mayor vessel internal iliaca artery bilateral by the anterior approach. A: Ligation of the left internal iliaca artery. B: Ligation of right internal artery. C: After ligation internal iliaca artery bilateral. 


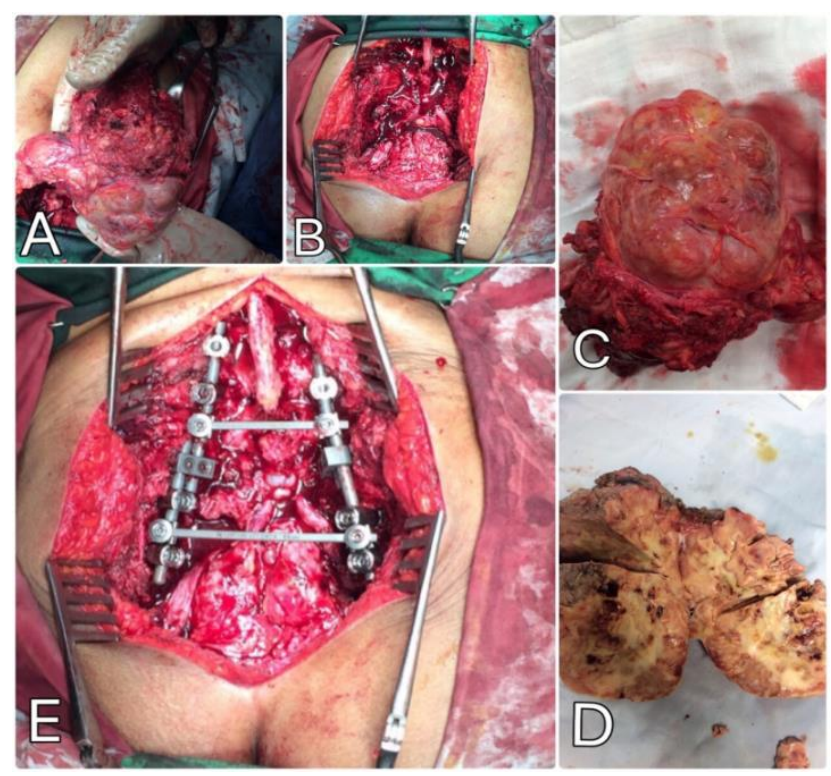

Fig. 3: Total sacrectomy and spinopelvic reconstruction. A: Totalsacrectomy. B: After a removal tumor. C\&D: Tumour. E: spinopelvic reconstruction.

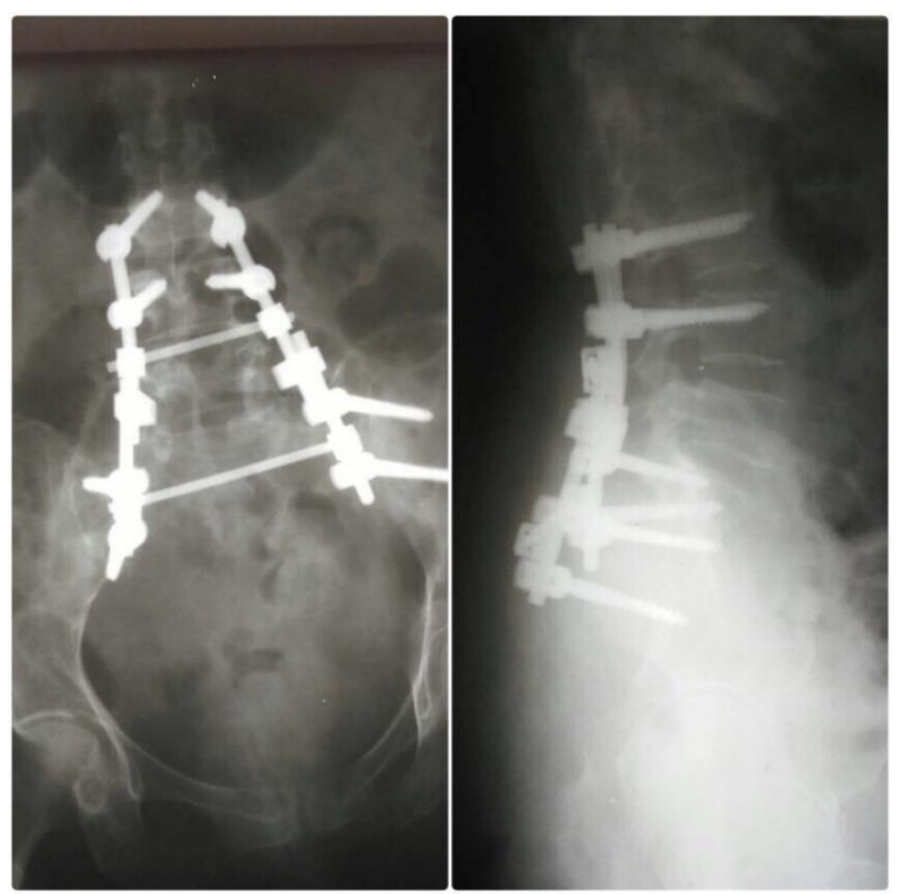

Fig. 4 : X rays post spinopelvic reconstruction. (left) Anteroposterior X-ray at 1 day after total sacrectomy and stabilization is achieved with 2 rods in order to provide stable fixation. (right) Lateral X-ray showing spinoiliac reconstruction. 


\section{Discussion}

\subsection{Management strategies}

GCT is locally aggressive. GCT is a benign bone tumor that often experiences local recurrence but is rare for distant metastases. Sacral GCT grows slowly, so it does not cause special symptoms. Local pelvic pain is a frequently expressed complaint that is often considered sciatic pain. Therefore the diagnosis of GCT is often late and is found with extensive invasion of the bones and sacral nerves root. The presence of a pelvic mass is also a symptom in patients who cause to come to health care. Investigations such as MRI and CT scans are needed to determine the location, size, and extent of GCT which will be a consideration for management. A fineneedle aspiration biopsy is an effective tool for the differential diagnosis of sacral GCT, aneurysmal bone cyst, giant cell reparative granuloma, chondroblastoma and brown tumor of hyperparathyroidism $[1,2,4]$.

\subsection{Surgical principles}

The standard treatment for the GCT is curettage and placement of a bone graft or bone cement. Recurrence rates have been reported to be as high as $50 \%$ or greater when the intralesional resection is not carefully performed. Follow-up treatment for recurrent disease consists of an aggressive resection and reconstruction involving a large osteoarticular allograft, cementation, endoprosthesis, or an excision-induced arthrodesis [1],[3],[4].

The concept of management tumors sacral regional with en block resection was introduced by senior and gunterberg in the 1970s. In certain patients, the act of sacrectomy can be the procedure of choice to reduce local recurrence and improve the survival of patients with complications of neurological disorders and sexual dysfunction. Operations are carried out by forming a team consisting of orthopedic oncology, general surgery, and spine surgery depending on the extent of the resection. Sacral GCT is hypervascularity so Preoperative embolization should be considered. Surgical actions with a combination of anterior-posterior approaches can be performed on the tumor with anterior expansion or involvement of the S-1 and S-2 segments. Reconstruction and stabilization using spinal instrumentation and an allograft or prosthesis may be done after total sacrectomy. Transperitoneal anterior approach as an alternative to the ilioinguinal approach, which provides simultaneous visualization of the anterior and posterior sacrum when combined with a posterior midline approach. Owing to the development of more vigorous surgery which enables wider oncological margins, local control of the tumors has significantly established. Sacrectomy following four surgical approaches; i.e., a combined sequential anterior and posterior approach, a combined simultaneous anterior and posterior approach, a posterior approach and combined posterior with extended ilioinguinal approaches. Variable approaches can be used in surgical techniques to remove the tumor such as anterior, posterior or combination of both. Used approach to get the optimal wide surgical margin. The posterior approach is mostly applicable for lesions below S3 level. These advantages are a single operation, shorter operative time, and less morbidity. Disadvantages include a potential for hemorrhage and possible violation of the pelvic viscera or ureters during removal tumor. At some centers, a simultaneous anteroposterior approach is preferred to visualize the anterior structures during osteotomy. The combined approach prefer for above S3 level. The anterior approach allows exposure of the entire sacrum with the mobilization of the rectum, ureter, and major vessels [1],[2],[3]. 
The operative approach to the sacral tumor is tailored to lesion size and relationship to the sacrum, sacroiliac joints, and sacral nerve roots. Combined anterior-posterior approaches may be required in some circumstances. Lumbopelvic reconstruction with instrumented fusion is recommended in cases involving the majority of the sacroiliac joint, or when a total or high sacrectomy is performed and can be achieved using the modified Galveston technique. Disconnection of the anococcygeal ligament and safe dissection of the tumor from the ventral pelvic structures is often aided by collaboration with a colorectal surgeon. Postoperative complications related to pressure-dependent wound and breakdown and infection are a major source of morbidity following sacrectomy, and therefore, collaboration with plastic surgery for layered wound closure with or without a flap is beneficial [3]. We did ligation of the internal iliac arteries by the anterior approach that may have helped reduce bleeding during mobilization of the specimen from posteriorly and following by spinopelvic reconstruction procedure.

In one study, blood loss is a significant consideration for these operations, with a mean blood loss of 9,276 mL reported in a series of 24 patients. With ligation of internal iliaca artery bilateral, it can decrease to $4,637 \mathrm{~mL}$. It was the same found in our case. Interoperative bleeding was about $4.500 \mathrm{~mL}$.

Surgical complications, such as superficial or deep wound infections or neurological deficits, cause significant morbidity after these complex operations. The patient experienced a superficial infection after surgery was treated successfully with serial debridements and parenteral antibiotics. ${ }^{1}$

\subsection{Neurological outcomes}

Neurological disorders after total resection produce morbidity associated with motor function, bowel, and bladder. Preoperative function and the level of sacrectomy greatly determine the risk of postoperative dysfunction. The involvement of the S2 nerve roots and lower can provide postoperative urinary and bowel dysfunction. The incidence of bowel and bladder dysfunction after high-level sacrectomy reaches $100 \%$ and decreases to $75 \%$ and $12.5 \%$ at lower levels. The incidence of bowel and bladder dysfunction after total sacrectomy involving resection of S1 roots are reaching $100 \%$. Resection of S1 nerve roots can provide the incidence of inability plantar flexion postoperative, and $40 \%$ of patients need ankle orthosis for mobilization. $100 \%$ bowel and bladder dysfunction reported after bilateral resection of S2-S5. Bilateral S2 preservation provides $40 \%$ and $25 \%$ of bowel and bladder function, respectively and increases to $100 \%$ and $69 \%$ when preserving S2 and S3 roots. Unilateral nerve root preservation is related with improved neurological function and recovery with a return of function at after six to eight months. Unilateral preservation of S3 provides $67 \%$ and $60 \%$ chance of complete bowel/bladder function suggesting that a majority of patients can maintain an optimal quality of life post sacrectomy [3].

\subsection{Radiotherapy and Chemotherapy}

Radiotherapy is used as one of the therapeutic modalities in GCT. Local recurrence and postradiotherapy complications such as fibrosis and the emergence of malignancy are risks of radiotherapy. Local recurrence rate reached $49 \%$. Radiotherapy should be used in conditions where the benefits and risks that might occur have been considered [1],[2]. The estimated frequency of malignant transformation of GCTs is $<1 \%$ but, after irradiation, it is as high as $10 \%$. Result study by Chakravarti reported 2 cases progressively in 5 to 8 months after being given radiotherapy at a dose of 40-70Gy and both required surgery. The remaining three patients 
were disease-free throughout follow-up (between 3 and 10 years). ${ }^{1}$ A dose of 50 Gy was recommended in order to optimize local control. Leggon et al. reported a $49 \%$ rate of local recurrence in patients with pelvic or sacral GCT treated with radiotherapy alone and an $11 \%$ risk of radiation-induced sarcoma, but Chakravarti et al. found only a 3\% risk. Improvements in radiotherapy targeting, in particular, the use of intensity modulated radiation, may prove beneficial for these tumors. Chemotherapy is not often used in the treatment of GCT, and no standard protocol exists for these tumors. Chemotherapy agents, including methotrexate, adriamycin, and cyclophosphamide, which are used in the treatment of sarcomas, are sometimes used for GCT. Adriamycin as Adjuvant chemotherapy may be effective in controlling local lesions.

\section{Conclusions}

Management sacral tumors with surgery in either total or partial sacrectomy with stabilization of the lumbopelvic shows satisfactory results and minimal complication rates. Sacral nerve observation during surgery is an important key to maintaining neurologic function after surgery. To reduce the risk of bleeding, preoperative embolization and iliac blood vessel ligation can be performed.

\section{References}

[1] CC Shen, H Li, ZL Shi, HM Tao and ZM Yang. Current Treatment of sacral Giant Cell Tumour of Bone: a Review. Department of Orthopaedics, Second Affiliates Hospital, School of Medicine, Zhejiang University, Hangzhou, China. The Journal of International Medical Research. 2012;40:415-425.

[2] Khodamorad jamshidi,Abolfazi Bagherifard, alizera Mirzaei, Mehrdad Bahrabadi. Giant Cell Tumor of the Sacrum : Series of 19 Patients and Review of the Literature. Shafa Orthopaedic Hospital, Iran University of Medical Science, Tehran. Iran. Arch Bone Jt. Surg. 2017;5(6):443450 .

[3] Michelle JC, Frank DV.Spinal Tumor Surgery:sepis Management and the Avoidance of Complications. Cancer Control April 2014, Vol. 21, No. 2. Available from: http ://moffitt.org

[4] Ming Zhou, Huilin Yang, Kangwu Chen, et al. Surgical Treatment of Giant Cell Tumor of the sacrum and spine combined with pre-operative transarterial embolization. Department of orthopedics Surgery. The first Affiliated Hospital of Soochow University, Suzhou, Jiangsu, Cina. Oncology Letter 6 : 185-190.2013. 